\title{
FLOW PATTERN ALTERATION NEAR A HYDROFOIL DUE TO EFFECTIVE SLIP: AN EXPERIMENTAL STUDY
}

\author{
SALIL GOGTE $^{1,2}$, ANDREA MAMMOLI $^{1}$ \& PETER VOROBIEFF ${ }^{1}$ \\ ${ }^{1}$ Department of Mechanical Engineering The University of New Mexico Albuquerque, New Mexico 87131, USA. \\ ${ }^{2}$ EcoMetric Consulting, 504 Windsor Way, Chester Springs, PA 19425, USA.
}

\begin{abstract}
We present an experimental investigation of water flow around a hydrofoil with a superhydrophobic patterned surface. The experimental setup uses a water tunnel to measure the drag over the hydrofoil and acquire velocity field measurements using particle image velocimetry (PIV). Drag reduction on the order of $10 \%$ or higher was observed on hydrofoils with irregular surface textures combined with super-hydrophobic coating, leading to effective Navier slip on the surface. However, we report that other macroscopic flow characteristics, including the stall angle, are also changed by application of the coating.
\end{abstract}

Keywords: drag reduction, experiment, particle image velocimetry, stall, superhydrophobic surface.

\section{INTRODUCTION}

Drag in fluids is a result of friction that opposes the motion of an object through the fluid or of the fluid through a device. Drag reduction would benefit a wide variety of applications, however, to date there are few demonstrated methods for reducing drag in laminar flows, although proved methods exist for fully developed turbulent drag reduction [1]. The laminar drag reduction has implications for confined biological systems [2, 3], permeability of porous media [4], lubrication of nano-machines [3], and in the microscale control of fluid flow. Moreover, it has been demonstrated that microscale changes in surface properties can have a macroscale effect on the flow manifesting in the laminar flow that can persist into the turbulent regime as well $[5,6]$.

In this paper, we discuss both laminar and turbulent drag reduction over Joukov-sky hydrofoils covered with microtextured superhydrophobic $(\mathrm{SH})$ surfaces, the characteristic surface feature sizes being much greater than the roughness associated with the SH coating itself. The effects are not limited to drag reduction, however: the macroscopic flow pattern itself is notably altered, for example, the stall zone over the hydrofoil is appreciably reduced in the case of the flow with effective slip.

The repulsion of water molecules over SH patterned surfaces is manifested by the reduction of surface energy, leading to the fluid maintaining contact with only a part of the surface. Due to the smaller contact area of the liquid on such surfaces, both the chemical reactions and bonding between the fluid and the surface are reduced [2]. Superhydrophobicity of a surface is characterized by a high contact angle 6 , defined as the angle formed by a liquid drop on a flat surface at the three-phase boundary, where the liquid, the solid, and surrounding gas (usually air) intersect. This angle can be determined by drawing a tangent to the fluid surface at the three-phase intersection. Surfaces with low $\left(\theta<90^{\circ}\right)$ contact angles, e.g., clean glass, are hydrophilic. If 6 is $90^{\circ}$ or greater, the surface is referred to as hydrophobic, with surfaces characterized by $6>140^{\circ}$ usually called superhydrophobic. One method of forming SH surfaces is the deposition of a chemically proportionate mixture of tetramethylorthosilicate and trifluoropropyltrimethoxysilane on the surface [7]. This method only works id a chemical bond can form between the surface and the coating materials. Patterned SH surfaces have 
motivated new research in the area of microfluidic devices owing to the presence of macroscopic effective slip [8], although there is no evidence of such a phenomenon near an unpatterned SH surface, where the largest features are nanometer-sized.

One could argue that the no-slip boundary which is accepted "by default" in fluid dynamics is in fact merely a good approximation. In reality, the fluid always slips at the wall - at least on the molecular level. This slip may play a role in devices that operate at nanoscales. Historically, the concept of slip boundary condition was proposed by Navier [9] almost 200 years ago. A schematic diagram of Navier's model is shown in Fig. 1. In this model the slip at the wall $u$ is proportional to the shear rate experienced by the fluid at the wall

$$
u=b \frac{\partial u}{\partial y}
$$

where $b$ is the slip length. Thus a non-zero slip velocity exists at the wall for finite values of $b$. Large slip lengths of $b>100 \mu \mathrm{m}$ have been reported for non-Newtonian fluids [10], however for simple Newtonian fluids such as pure water flowing past smooth hydrophobic surfaces, much smaller (nanoscale) slip lengths have been observed [2].

In an assessment of relative importance of surface roughness and fluid-surface intermolecular interactions in determining the boundary condition [8], the critical shear stress and shear rate were found to increase nearly exponentially with increasing roughness and diverge at 6 $\mathrm{nm}$ rms roughness, thus deviating from the predictions using the no-slip boundary condition. Thus the local intermolecular interactions dominate when the surface is very smooth, but roughness dominates otherwise. For Newtonian fluids between vibrating surfaces, the no-slip condition was verified at lower flow rates (peak normalized by surface-to-surface spacing), but partial slip was implied when the flow rate exceeded a certain critical level [8]. This change was directly related to the contact angle (surface wettability). With increasing flow rate and partially wetted surfaces, the observed hydrodynamic forces were up to 2-4 times smaller than those that the no-slip boundary condition would imply. The experiments presented in this paper show that reduced hydro-dynamic forces are observed on hydrofoils (as

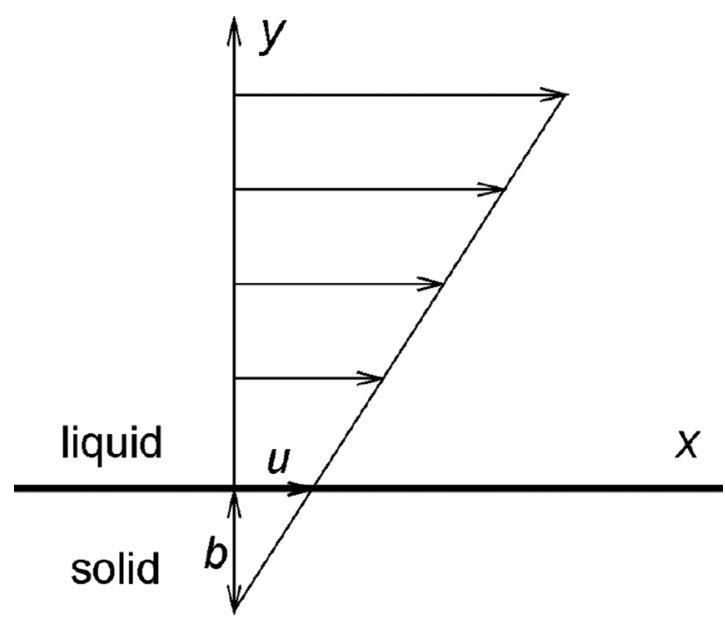

Figure 1: Schematic diagram defining the slip velocity $\mathrm{u}$ and slip length $\mathrm{b}$ at the solid-liquid interface. 
compared to the no-slip boundary condition) in the presence of SH coating applied to surfaces with characteristic roughness on the order of $10 \mu \mathrm{m}$. We also investigate the effect of varying roughness and surface structure on the superhydrophobicity.

The nature of fluid-surface interactions near a textured SH surface is illustrated in Fig. 2. The characteristic size of the roughness ideally should be on the same order of magnitude as the boundary layer thickness in external flow, but much less than the capillary length $(2.7 \mathrm{~mm}$ for water). The surface can be patterned with regular/ordered roughness (e.g., grooves) or irregular/random roughness (random peaks). The effective contact area between the fluid and the surface is reduced due to the low surface energy, resulting in large interfacial tensions and formation of fluid-free surface areas. These areas exist because there is insufficient energy to deform the fluid boundary to bring it in contact with the entire surface (Fig. 2b). This will lead to a velocity profile in the idealized fluid-surface contact plane with zero velocity in the contact areas and nearly parabolic velocity distribution in the areas of no contact. A velocity average at the nominal fluid boundary (contact plane) would be greater than zero (Fig. 2c). Similar behavior can be expected for an irregular texture (Fig. 2d).

Our earlier work $[5,6]$ compared the behavior of droplets rolling down a textured SH surface and the drag on a hydrofoil with the same surface treatment applied. We found consistent results strongly suggesting effective slip with effects noticeable on the macroscopic scale, and persisting into the turbulent regime, with Reynolds number (based on hydrofoil chord length) up to 11,000 [5].

Interestingly, in the following ten years there have been multiple studies of flow behavior near various surfaces with effective slip (see Refs. [11, 12]) for reviews), relatively little

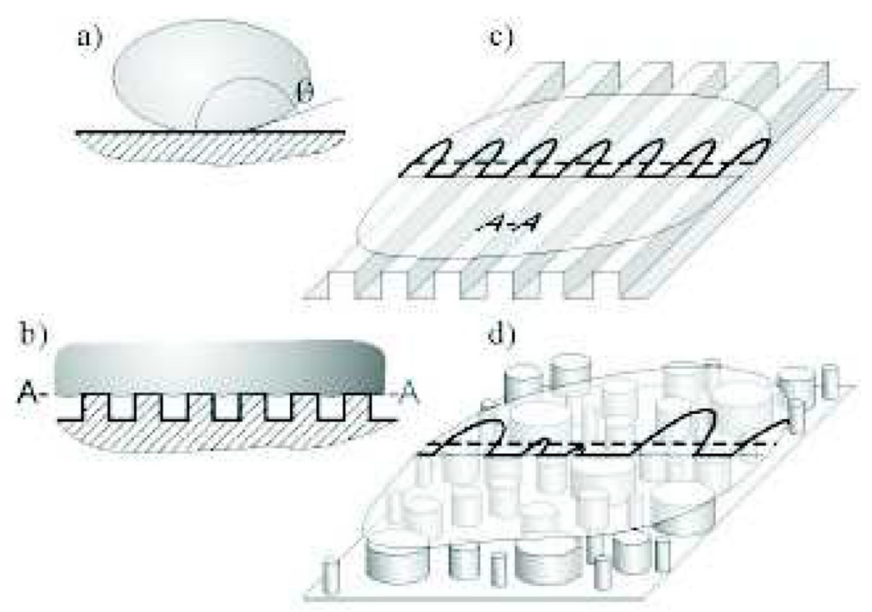

Figure 2: Schematics of fluid behavior near a superhydrophobic surface: a - side view of a droplet on smooth surface with contact angle 6 shown, $b$-cross-section of a grooved surface with fluid on top and air trapped in the grooves and A-A indicating the location of the plane of the nominal fluid boundary, $\mathrm{c}$ - perspective view of the grooved surface showing fluid velocity profile in the A-A plane with the dashed line indicating average (i.e., slip) velocity, $\mathrm{d}$ - perspective view of an idealized irregularly textured surface showing the fluid velocity profile and effective slip velocity at the fluid boundary. Partially based on [5]. 
attention has been paid to how the introduction of effective slip changes the flow patterns, in particular, in external flow. Experimental works on the subject have been especially scarce. Effective slip has been shown to affect lifting surface [13] and cylinder wakes [14]. Turbulent drag and turbulence fluctuation intensity have been measured in the boundary layer near a superhydrophobic surface, with a significant reduction in both [15].

In the following narrative, we compare earlier measurements of drag on the hydrofoil with velocity-field measurements that confirm the conclusion that laminar drag reduction is achieved via the surface treatment that produces a non-trivial effective slip velocity on the hydrofoil surface. We also discuss and explain the effects of effective slip boundary condition on the flow pattern observed around the hydrofoil, including reduction in the size of the stall area.

\section{EXPERIMENTAL SETUP}

The water tunnel experimental setup shown in Fig. 3 is used to measure hydrodynamic forces over symmetric Joukovsky hydrofoils. The hydrofoils are initially set at a $0^{\circ}$ angle of attack to eliminate the lift component of the hydrodynamic force. The arrangement uses a two-component dynamometer capable of measuring forces between 0.5 and $50 \mathrm{gf}$ (gram-force). The measurements are facilitated with a linear variable differential transducer (LVDT), capable of measuring bi-directional deflections, the analog output of which is fed to a voltmeter. The voltmeter sensitivity is adjusted in correlation with the measurement range of the drag forces, appropriate consideration being given to the noise effect. The water velocity and hence the Reynolds number of the flow through the test section is varied by changing the driving frequency of the recirculating pump. The dimensions of the test section of the tunnel are $L=43 \mathrm{~cm}, W=15 \mathrm{~cm}$, and $H=14 \mathrm{~cm}$. The hydrofoil is mounted on the dynamometer strut at mid-span of the test section.

The equation for the Joukovsky hydrofoil profile is

$$
z=\zeta+\frac{c^{2}}{\zeta},
$$

where $\mathrm{c}$ is a scalar constant which transforms a cylinder in the complex plane $\zeta$ into a hydrofoil in the complex plane $z$ [16]. The characteristics of the hydrofoil can be set via the offset of the cylinder center in the $\zeta$ plane from the origin.

The actual size is obtained by scaling the numerical output to obtain the desired coordinate dimensions. The coordinates, after proper scaling, are used to generate the profile plot on a defined coordinate system as shown in Fig. 4a and b shows the prototype model generated from the profile plot at the desired span length. ABS and UV plastic hydrofoils are fabricated from the models with rapid prototyping techniques.

The results presented in this paper are for the water flow past a Joukovsky hydrofoil with a chord length $l=4.3 \mathrm{~cm}$, span $3.2 \mathrm{~cm}$, and thickness $25 \%$ of the chord. The freestream velocity $U_{\infty}$ is varied to produce a range of Reynolds numbers $R e=U_{\infty} / \mathrm{v}$ from 1,500 to 11,000 . The experiments were conducted with hydrofoils subjected to several types of surface treatment. We investigated two types of smooth surfaces - ABS plastic with and without the SH coating, and several types of textured coating produced by pasting sandpaper (with or without SH coating) over the hydrofoil surface. 2,500 and 1,200 grit sandpaper was used, with the corresponding characteristic feature sizes being 8 and $15 \mu \mathrm{m}$.

$\mathrm{SH}$ coating was prepared by a variation of the low temperature/pressure aerogel thin film process [7] wherein tetraethylorthosilicate was replaced with a 1.0:0.3 molar ratio of 


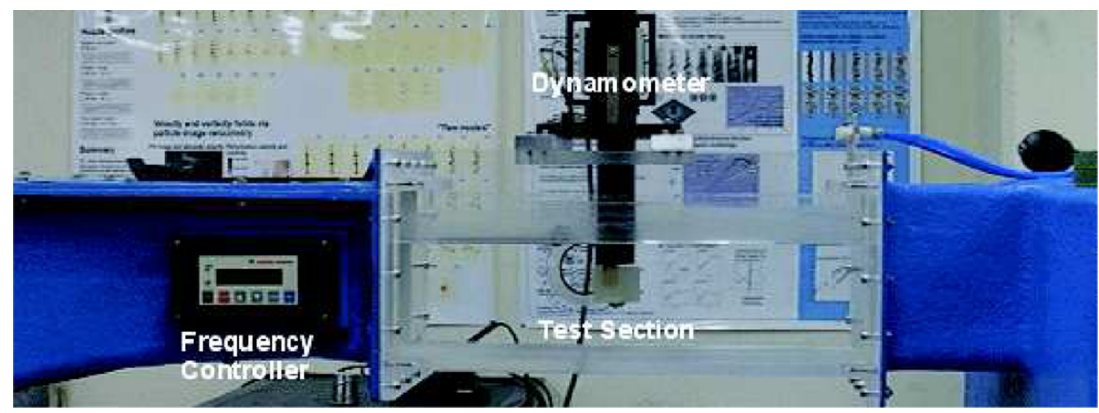

Figure 3: Side view of the water tunnel with the hydrofoil mounted on the dynamometer strut. Flow direction is from left to right.
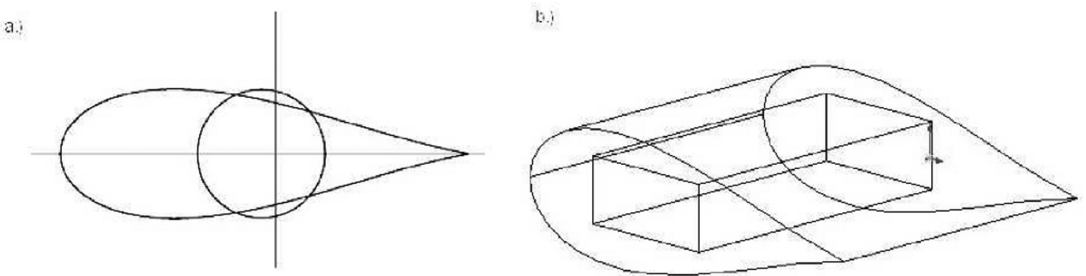

Figure 4: Line diagrams of Joukovsky hydrofoil used in experiment: a - Joukovsky profile plot, $\mathrm{b}$ - perspective view. Rectangular hole is for dynamometer strut mount, thus when installed in the water tunnel, the hydrofoil is oriented with the spanwise direction straight down.

tetramethylorthosilicate and trifluoropropyltrimethoxysilane. Drying of the aerogel produces a fractally rough surface with nanometer-size features terminated with trimethylsilyl and trifluoropropyl ligands. This coating process can be applied to many materials. The thickness of the coating is about half a micron.

On the plain acrylic surface with the $\mathrm{SH}$ coating, the advancing and receding contact angles measured were $149.4^{\circ}$ and $148.8^{\circ}$ correspondingly, while on the textured SH surface ( $8 \mu \mathrm{m} \mathrm{rms}$ roughness) the angles measured were $169.2^{\circ} / 170.4^{\circ}$. Some preliminary experiments were conducted on grooved acrylic SH coated hydrofoils, and the contact angles recorded were $169.2^{\circ} / 168.2^{\circ}$. The $\mathrm{SH}$ coating was also tested on diamond lap film and overhead transparency film where the contact angles measured were $170.8^{\circ} / 169.2^{\circ}$ and $157.8^{\circ} / 158.5^{\circ}$ respectively.

Prior to any drag measurements the tunnel was calibrated and a suitable range of measurements, in terms of the Reynolds numbers, was established for conducting experiments. The dynamometer was mounted on a leveled support with the deflections of the strut constrained to the horizontal in order to eliminate the vertical lift forces. The deflections of the strut and the corresponding changes in the voltmeter reading, were observed for forces between $1 \mathrm{gf}$ and $10 \mathrm{gf}$. The dynamometer was equipped with ten spans of measurement, span 9 being the least sensitive while span 0 being the most sensitive. The choice of the span being used was dependent on the range of drag forces being measured. The water velocity in the test section of the tunnel was independently measured at several motor frequencies using the Particle Image Velocimetry (PIV) setup described in the next paragraph. 
For the external flow it is highly desirable to obtain the velocity profiles in the boundary layer, especially near the leading edge of the hydrofoil, where the boundary layer is thinnest and most of the drag accumulates. To resolve the velocity fields near the uncoated and $\mathrm{SH}$ coated hydrofoils, we performed a series of PIV measurements. The custom-built PIV arrangement uses a double-pulsed Nd:YAG laser illuminating the flow with a light sheet of $0.2 \mathrm{~mm}$ thickness. The images were acquired with a $2048 \times 2048$ pixel 12-bit digital camera. The delay between the exposures can be varied to achieve optimal results during the velocity field acquisition. Figure 5 shows PIV images for the uncoated and $8 \mu \mathrm{m}$-texture coated hydrofoils at $R e=7,300$. For these images, the delay between the exposures was $2 \mathrm{~ms}$ and the pixel resolution was 18 microns. The tracer used in the experiment was titanium dioxide (characteristic particle size 0.2 microns). A standard cross-correlation PIV algorithm [17] with a window size of $32 \times 64$ pixels was used to acquire the velocity vectors on a regular grid. A conservative estimate of the PIV velocity measurement accuracy for this case [18] produces an error estimate not exceeding $1.2 \mathrm{~mm} / \mathrm{s}$, or $0.6 \%$ of the freestream velocity.

\section{RESULTS AND ANALYSIS}

Drag measurements for the Joukovsky hydrofoils were earlier made for the following surface types: $\mathrm{SH}$-coated textured surfaces with $15-\mu \mathrm{m}$ and $8-\mu \mathrm{m}$ roughness, the same rough surface without the coating and smooth ABS and Polyjet UV cured surfaces [5]. Highest drag reduc-
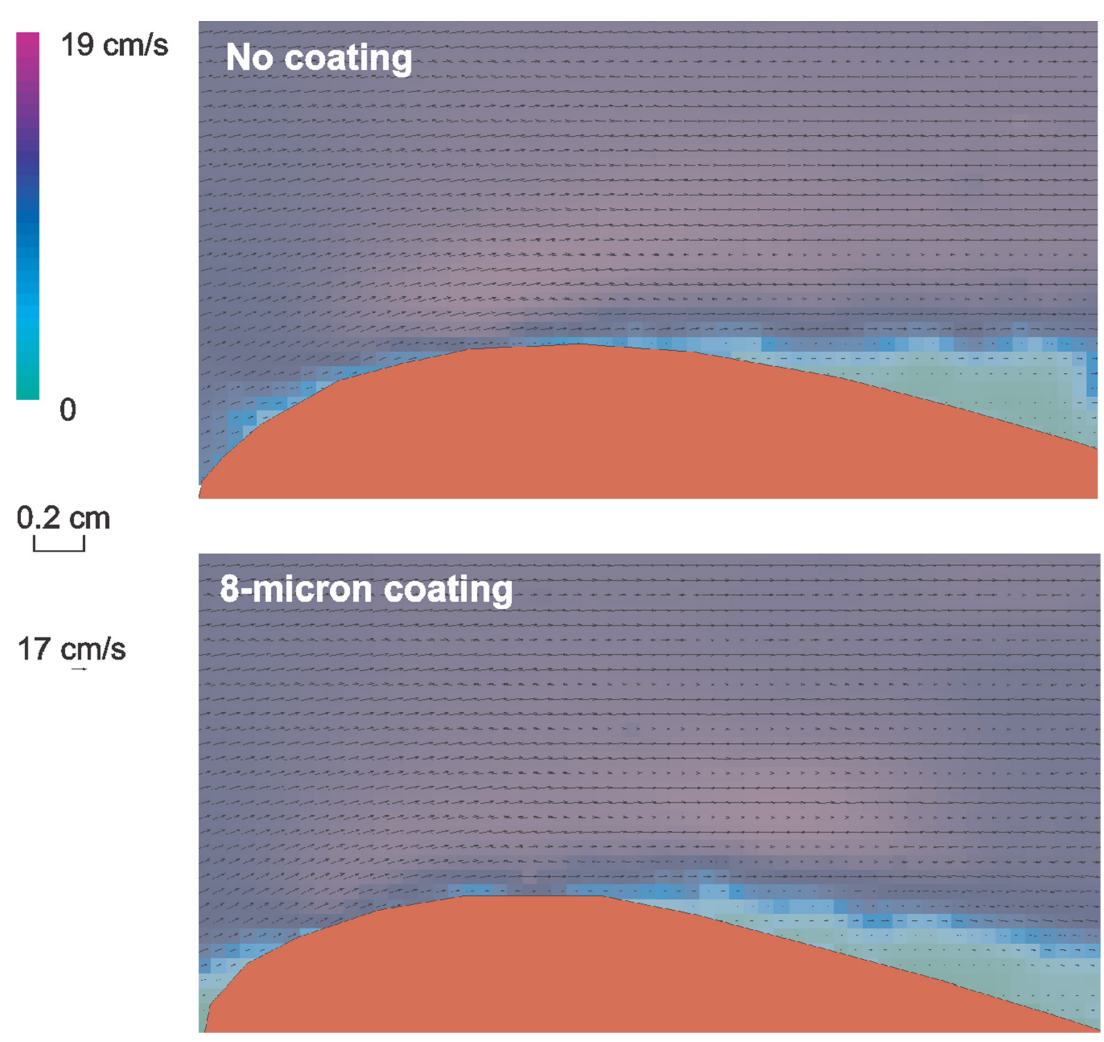

Figure 5: Instantaneous velocity fields acquired near a smooth uncoated (top) and textured and coated (bottom) hydrofoil at $R e=7,300$. 
tion (18\% compared with the smooth uncoated hydrofoil) was observed with the $8 \mu \mathrm{m}$ textured SH surface at $R e=1,500$. This was consistent with our predictions from the numerical simulations [5] and also the results obtained by measuring the velocities of droplets rolling down inclined flat surfaces with the same coatings. The drag coefficients on the Reynolds number of the flow for each surface type, along with the percentage of drag reduction for the hydrofoils with textured surfaces compared with the smooth UV plastic hydrofoils (SH coated). The drag coefficient $C_{\mathrm{D}}$ represents the normalized drag force $D$ :

$$
C_{D}=\frac{2 D}{p U_{\infty}^{2} S},
$$

where $S$ is the cross-sectional area of the hydrofoil in the direction of the freestream. It was also observed that as Re increases, the drag reduction is lowered ( $7 \%$ at $R e=11,000)$. Notably, the textured surface without the SH coating was characterized by a several percent increase in drag compared with the smooth uncoated surface.

This earlier work suggested that the main region of drag reduction due to effective macroscopic slip on the boundary is the leading-edge region of the wing, where the boundary layer is the thinnest and the effect of macroscopic slip on shear in the flow would be the most prominent. The PIV measurements presented here (Fig. 5) are still insufficiently highly resolved to directly assess the velocity profiles near the leading-edge in the boundary layer. However, it is noteworthy that the coating apparently has an effect on the boundary-layer thickness farther downstream (last third of the chord length), reducing the extent of the sep-

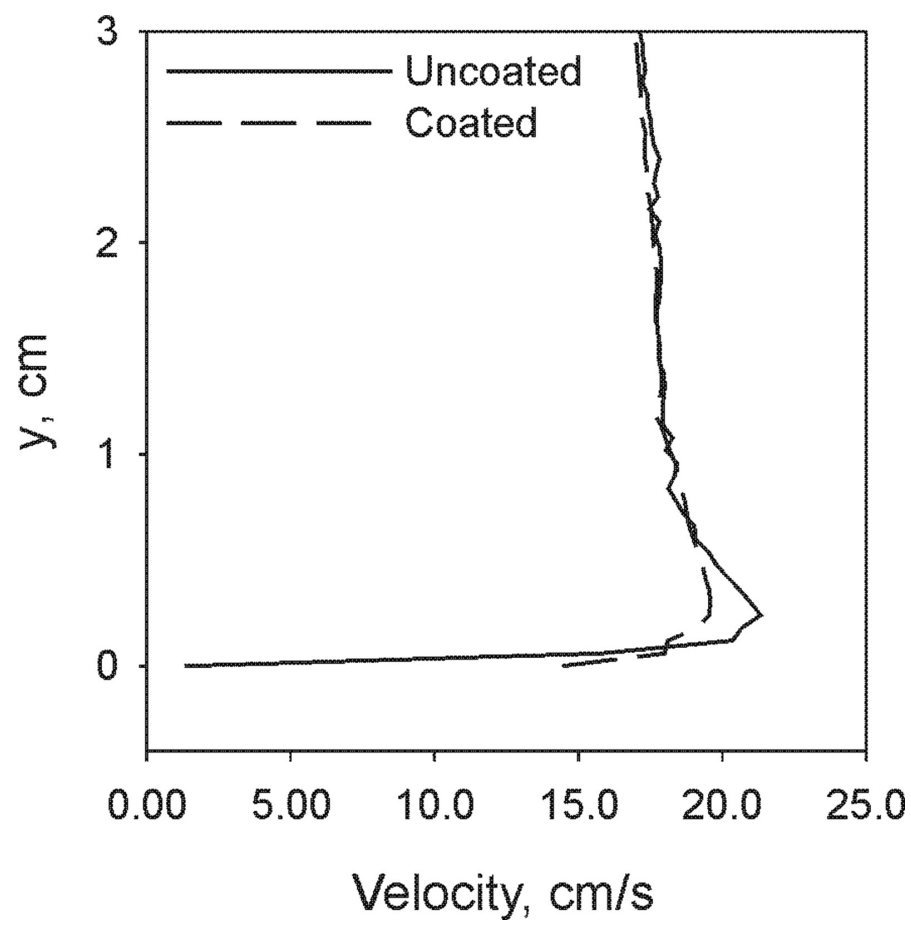

Figure 6: Instantaneous velocity profiles at the point of maximum hydrofoil thickness for uncoated $\& 8 \mu \mathrm{m}$ textured, SH coated hydrofoils. $R e=7,300$. 
aration zone compared with the uncoated case. The onset of separation is apparently retarded in the presence of the coating, although more study is necessary to put quantitative bounds on this effect.

We also compared the velocity profiles at the point of maximum thickness for the coated ( $8 \mu \mathrm{m}$ texture) and uncoated hydrofoil (Fig. 6). While the overall shape of the profiles faithfully reproduces the actual flow features, the effective slip velocity value of $14.6 \mathrm{~cm} / \mathrm{s}$ for the coated surface is an artifact of the PIV interrogation due to insufficient resolution in the boundary layer. However, manual particle tracking in the immediate vicinity of the boundary at the point of maximum thickness still produces an appreciable non-zero velocity in the range from 1 to $3.6 \mathrm{~cm} / \mathrm{s}$. These values are actually quite consistent with the effective slip velocities one could infer from the drag reduction measurements [5].

\section{CONCLUSIONS}

The flow-field measurements near a hydrofoil with superhydrophobic coating applied to a textured substrate are consistent with earlier drag measurement results. We observe an appreciable drag reduction and corresponding changes in the velocity field when we compare the flow near a smooth hydrofoil with that near a textured and SH-coated hydrofoil of the same outside dimensions. The physical mechanism responsible for this drag reduction (reduction in fluid-solid contact area) has also been demonstrated to work for drops rolling down an inclined surface, thus inspiring hope that drag-reduction techniques based on the combination of surface texturing with superhydrophobic coatings will be applicable to a wide variety of flows.

Most importantly, it is demonstrated that application of a superhydrophobic coating to a hydrofoil can not only reduce drag, but affect the macroscopic characteristics of the surrounding flow, leading to significant changes of the stall zone geometry. In principle, stall on the lifting surfaces due to boundary layer separation is an inherent feature of viscous flow, notably absent in ideal-flow theory. Accordingly, by using effective-slip surfaces (where the macroscopic boundary condition differs from no-slip) it may be possible to mitigate lift loss due to stall. Future studies are necessary to confirm this assertion.

\section{ACKNOWLEDGMENTS}

This work was supported in part by the US Department of Energy (DOE) office of Basic Energy Sciences, Division of Materials Science and Engineering, US Air Force Office of Scientific Research (AFOSR), and by Sandia National Laboratories Lab-Directed Research and Development (LDRD) program.

\section{REFERENCES}

[1] Jovanovic, J., Pashtrapanska, M., Frohnapfel, B., Durst, F., Koskinen, J. \& Koskinen, $\mathrm{K}$., On the mechanism responsible for turbulent drag reduction by dilute addition of high polymers: theory, experiments, simulations, and predictions. Journal of Fluids Engineering, 128(1), pp. 118-130, 2006. http://dx.doi.org/10.1115/1.2073227

[2] Watanabe, K., Udagawa, Y. \& Udagawa, H., Drag reduction of Newtonian fluid in a circular pipe with a highly water-repellent wall. Journal of Fluid Mechanics, 381, pp. 225-238, 1999.

http://dx.doi.org/10.1017/S0022112098003747 
[3] Craig, V.S.J., Neto, C. \& Williams, D.R.M., Shear-dependent boundary slip in an aqueous Newtonian liquid. Physical Review Letters, 87(5), 054504, 2001.

[4] Barrat, J.L. \& Bocquet, L., Large slip effect at a nonwetting fluid-solid interface. Physical Review Letters, 82(23), pp. 4671-4674, 1999.

[5] Gogte, S., Vorobieff, P., Truesdell, R., Mammoli, A., Swol, F.V., Shah, P. \& Brinker, C.J., Effective slip on textured superhydrophobic surfaces. Physics of Fluids, 17(5), 51701, 2005.

http://dx.doi.org/10.1063/1.1896405

[6] Truesdell, R., Mammoli, A., Vorobieff, P., Swol, F.V. \& Brinker, C.J., Drag reduction on a patterned superhydrophobic surface. Physical Review Letters, 97(4), 044504, 2006. http://dx.doi.org/10.1103/PhysRevLett.97.044504

[7] Prakash, S.S., Brinker, C.J., Hurd, A.J. \& Rao, S.M., Silica aerogel films prepared at ambient pressure by using surface derivatization to induce reversible drying shrinkage. Nature, 374, pp. 439-443, 1995.

[8] Zhu, Y. \& Granick, S., Rate-dependent slip of Newtonian liquid at smooth surfaces. Physical Review Letters, 87(9), 096105, 2001. http://dx.doi.org/10.1103/PhysRevLett.87.096105

[9] Navier, C.L., Memoire sur les lois du mouvement des fluides. Mémoires de l'Academie Royale des Sciences de l'Institut de France, 6, pp. 389-440, 1823.

[10] Mhetar, V. \& Archer, L.A., Slip in entangled polymer solutions. Macro-molecules, 31(19), pp. 6639-6649, 1998.

[11] Rothstein, J.P., Slip on superhydrophobic surfaces. Annual Review of Fluid Mechanics, 42, pp. 89-109, 2010.

[12] Lee, T., Charrault, E. \& Neto, C., Interfacial slip on rough, patterned and soft surfaces: A review of experiments and simulations. Advances in Colloid and Interface Science, 210, pp. 21-38, 2014.

[13] Daniello, R., Valle, K.D. \& Rothstein, J., Slipping through the water: A study of superhydrophobic hydrofoils. In APS Meeting Abstracts, 1, p. 24007, 2012.

[14] Daniello, R., Muralidhar, P., Carron, N., Greene, M. \& Rothstein, J.P., Influence of slip on vortex-induced motion of a super-hydrophobic cylinder. Journal of Fluids and Structures, 42, pp. 358-368, 2013.

[15] Haibao, H., Peng, D., Feng, Z., Dong, S. \& Yang, Wu., Effect of hydrophobicity on turbulent boundary layer under water. Experimental Thermal and Fluid Science, 60, pp. 148-156, 2015.

[16] Panton, R.L., Incompressible Flow, John Wiley \& Sons, 2006.

[17] Westerweel, J., Fundamentals of digital particle image velocimetry. Measurement Science and Technology, 8(12), pp. 1379-1392, 1997.

[18] Prasad, A.K., Adrian, R.J., Landreth, C.C. \& Offutt, P.W., Effect of resolution on the speed and accuracy of particle image velocimetry interrogation. Experiments in Fluids, 13(2-3), pp. 105-116, 1992. 